\title{
Zum Gehalt an Seston und Plankton im tropischen Atlantik ${ }^{1}$
}

\author{
ERIK HAgMeIER \\ Biologische Anstalt Helgoland, Meeresstation, Helgoland
}

\begin{abstract}
On the content of seston and plankton in the tropical Atlantic. Some results of two cruises in 1963 during the International Cooperative Investigations of the Tropical Atlantic (ICITA) are reported and interpreted. The average vertical distribution gives seston (particulate matter) values from 20 to $250 \mathrm{mg} / \mathrm{m}^{3}$, and albumin equivalents from 2 to $28 \mathrm{mg} / \mathrm{m}^{3}$. These amounts are considerably less than those observed in the northern part of the Atlantic Ocean. A great variation was observed, which could not always be explained by the general circulation. Accumulation at the surface was more obvious than concentrations of seston and plankton near or in the discontinuity layer of density. A stimulating influence of the equatorial undercurrent on productivity could not be established. In most areas the standing stock of micro-plankton was found to be low when compared to the biomass of larger plankton organisms.
\end{abstract}

\section{EINLEITUNG}

\section{Die internationalen Untersuchungen im tropischen Atlantik}

Ende 1960 wurde die Intergovernmental Oceanographic Commission (IOC) von der UNESCO ins Leben gerufen. Als Ziel wurde ihr die Förderung wissenschaftlicher Forschungen zur Erkenntnis der Eigenschaften und Nahrungsquellen des Meeres gesetzt. Die ersten internationalen Untersuchungen, die von dieser Kommission organisiert wurden, waren die International Cooperative Investigations of the Tropical Atlantic (ICITA): Ein großes Gebiet sollte durch die Schiffe vieler Nationen synoptisch untersucht werden. Inzwischen ist der größte Teil des Programms erfolgreich abgelaufen. Von Februar bis Mai 1963 waren 13 Schiffe im tropischen Atlantik tätig (EQUALANT I); Wiederholungen erfolgten im Spätsommer 1963 (EQUALANT II) und mit etwas veränderter Fragestellung im Winter und Sommer 1964.

Untersuchungen über die Vertellung von Seston und Plankton wurden von fast allen beteiligten Schiffen ausgeführt mit vorher vercinbarten, gleichen Methoden. An der Auswertung des gewonnenen umfangreichen Materials wird noch gearbeitet. Vor einer später erst möglichen umfassenden Darstellung soll im folgenden schon mit den Ergebnissen von zwei Reisen des Verfassers die Größenordnung der angetroffenen Bestände umrissen werden.

\footnotetext{
${ }^{1}$ Herrn Professor Dr. Adolf Bückmann zum 65. Geburtstag in Verehrung gewidmet.
} 


\section{Zur Hydrographie des tropischen Atlantik}

Im größten Teil des durch die ICITA erfaßten Gebietes herrscht an der Meeresoberfläche ein Wassertransport nach Westen vor - angeregt durch die Passatwinde nördlich und südlich des Aquators. Unter der zwischen ihnen befindlichen Zone der Kalmen kann sich eine Ausgleichsströmung nach Osten entwickeln. Lage und Ausdehnung dieses äquatorialen Gegenstromes werden durch die etwas asymmetrische Lage
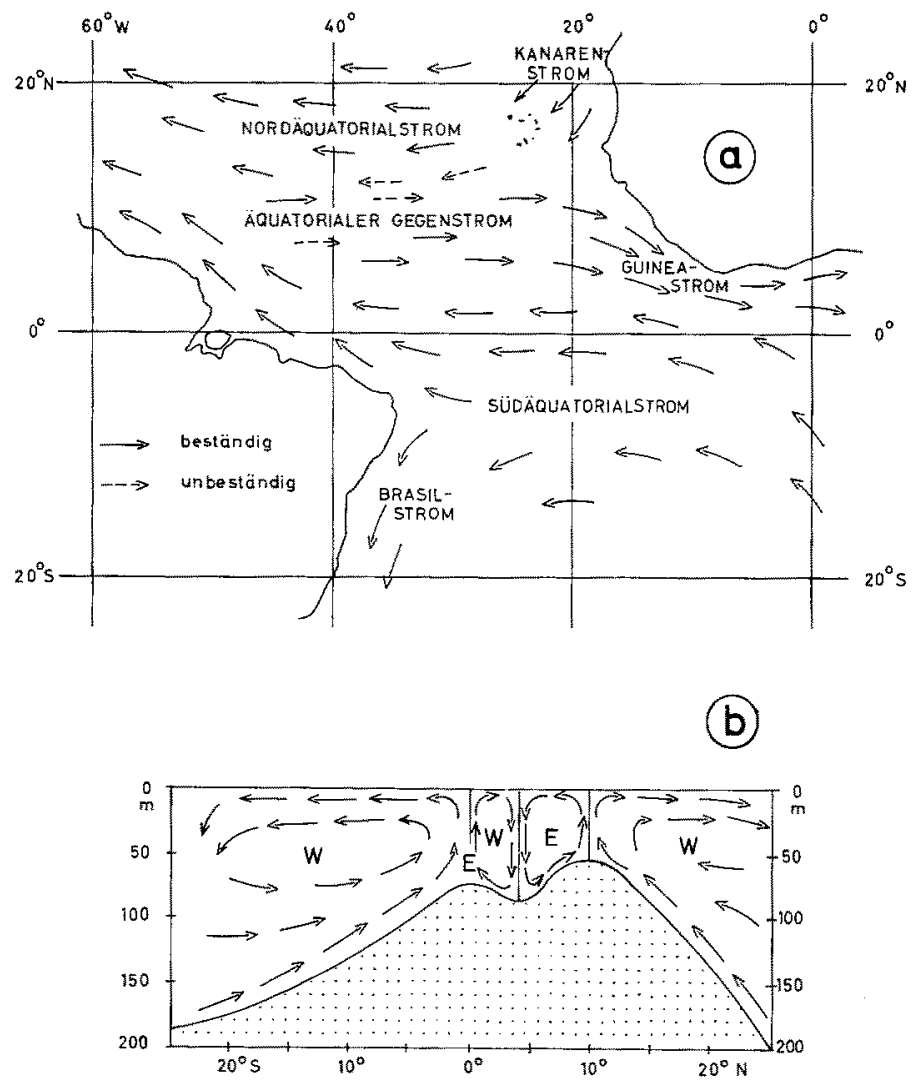

Abb. 1: Schematische Darstellung der Strömungen im tropischen Atlantik. a: Oberflächenströmungen im Nordsommer (nach ScHотт 1943), b: Querzirkulation in der Deckschicht (nach SVerdrup et al. 1942). W: Strömung nach Westen; $E$ : Strömung nach Osten. Unter dem Aqquator, in $60 \mathrm{~m}$ Tiefe, der Kern des Unterstromes $(E)$. Wasser unter der Temperatur-Sprungschicht punktiert

des Windfeldes zum Aquator und dessen Verschiebung zwischen Sommer und Winter bestimmt. Im Nordwinter können ostwärts gerichtete Strömungen bis etwa $25^{\circ} \mathrm{W}$ beobachtet werden; sie sind nicht wesentlich über den an der afrikanischen Küste von Nordwest nach Südost ziehenden Guineastrom ausgedehnt. Im Nordsommer aber erstreckt sich der Gegenstrom zwischen $5^{\circ}$ und $10^{\circ} \mathrm{N}$ über die ganze Breite des Ozeans 
bis $50^{\circ} \mathrm{W}$ (vgl. Abb. 1a, Schot't 1943 und die Karten des U.S. Navy Hydrographic OFFICE 1962).

Mit den Oberflächenströmungen sind Vertikalzirkulationen verbunden, Konvergenzen und Divergenzen, und eine unterschiedliche Tiefenausdehnung der warmen Deckschicht (Abb. 1b). Diese hydrographischen Gegebenheiten beeinflussen wiederum die Verteilung von Seston und Plankton.

Unsere besondere Aufmerksamkeit galt der Untersuchung des äquatorialen Unterstromes, dessen Vorkommen im Atlantik erst vor wenigen Jahren durch Messungen russischer und amerikanischer Forschungsschiffe bestätigt wurde. Unter einer westlichen Oberflächenströmung liegt in etwa $60 \mathrm{~m}$ Tiefe der Kern eines starken ostwärts gerichteten Stromes, der nur eine sehr geringe Ausdehnung nördlich und suidlich vom Aquator hat (Metcalf et al. 1962, Crease \& Pogson 1964). Am Cromwell-Strom des Pazifischen Ozeans wurde berechnet, daß die Reibung einen beträchtlichen Teil der Energie des Unterstromes verbraucht; daraus ergeben sich hohe Austauschwerte (WyrtKI \& BenNETt 1963). Im Atlantik wurde deshalb durch eine besonders gute Nährstoff-Versorgung der Oberschicht eine Erhöhung der Planktonproduktion am. Aquator erwartet.

\section{MATERIAL UND METHODEN}

\section{Die Herkunft der untersucbten Proben}

Die Reiserouten sind aus Abbildung 2 ersichtlich. Nach der Überfahrt der "Explorer" von Norfolk (Virginia) begannen die hydrographischen und biologischen Stationen vor Dakar. Auf drei Schnitte vor der afrikanischen Küste quer zum GuineaStrom folgte ein Schnitt auf dem 15. westlichen Längengrad von $4^{\circ} \mathrm{N}$ bis $15^{\circ} \mathrm{S}$. Besondere Messungen am Aquator galten der Erfassung des Unterstromes. Nach dieser ersten Beobachtungsperiode vom 16. 2. bis 7. 3. 1963 wurde Recife (Brasilien) angelaufen; auf dem Weg dorthin wurden Oberflächenproben genommen. Vom 20. 3. bis 9. 4. wurden die vorher ausgeführten Schnitte in umgekehrter Richtung wiederholt der Aquator-Schnitt auf $15^{\circ} 30^{\prime} \mathrm{W}$-, die letzte Station lag, wie die erste, vor Dakar. Die Lage der einzelnen Stationen ist auf den Abbildungen 4 bis 6 angegeben.

Die Stationen der "Casco" hatten nach dem Auslaufen aus Boston (Mass.) auf der Fahrt durch die Sargasso-See zunächst eintägigen Abstand. Auf $20^{\circ} \mathrm{N}$ begann mit engerer Stationsfolge der Schnitt über den Aquator; er wurde vom 31. 7. bis 14. 8. auf etwa $25^{\circ} \mathrm{W}$ bis $15^{\circ} \mathrm{S}$ ausgefuhrt. Die anschließende Fahrt nach Rio de Janeiro wurde zu einem Schnitt durch den Brasilstrom ausgenutzt. Auf der Rückreise über die Bermudas nach Boston wurden nur Oberflächenproben genommen.

\section{Die angewandten Methoden}

Zur Messung des Zooplankton-Bestandes hatte man vereinbart, die warme Oberflächenschicht bis zum Beginn des Temperaturabfalls während der Nachtstunden mit einem genormten Planktonnetz $\mathrm{zu}$ durchfischen. Dieses Netz hatte einen Offnungs- 
durchmesser von $1 \mathrm{~m}$, der Netzbeutel (Perlongewebe, Gaze 3, Maschenweite ca. $0,33 \mathrm{~mm}$ ) war etwa $4 \mathrm{~m}$ lang und endete in einem leicht abnehmbaren Plastikbecher. Im Offnungsring befand sich ein Propeller mit Umdrehungszähler zur Bestimmung des eingeströmten Wasservolumens. Auf den Fahrten der "Explorer" und "Casco" wurde gewöhnlich mit diesem Netz gearbeitet, während das Schiff auf einer hydro-

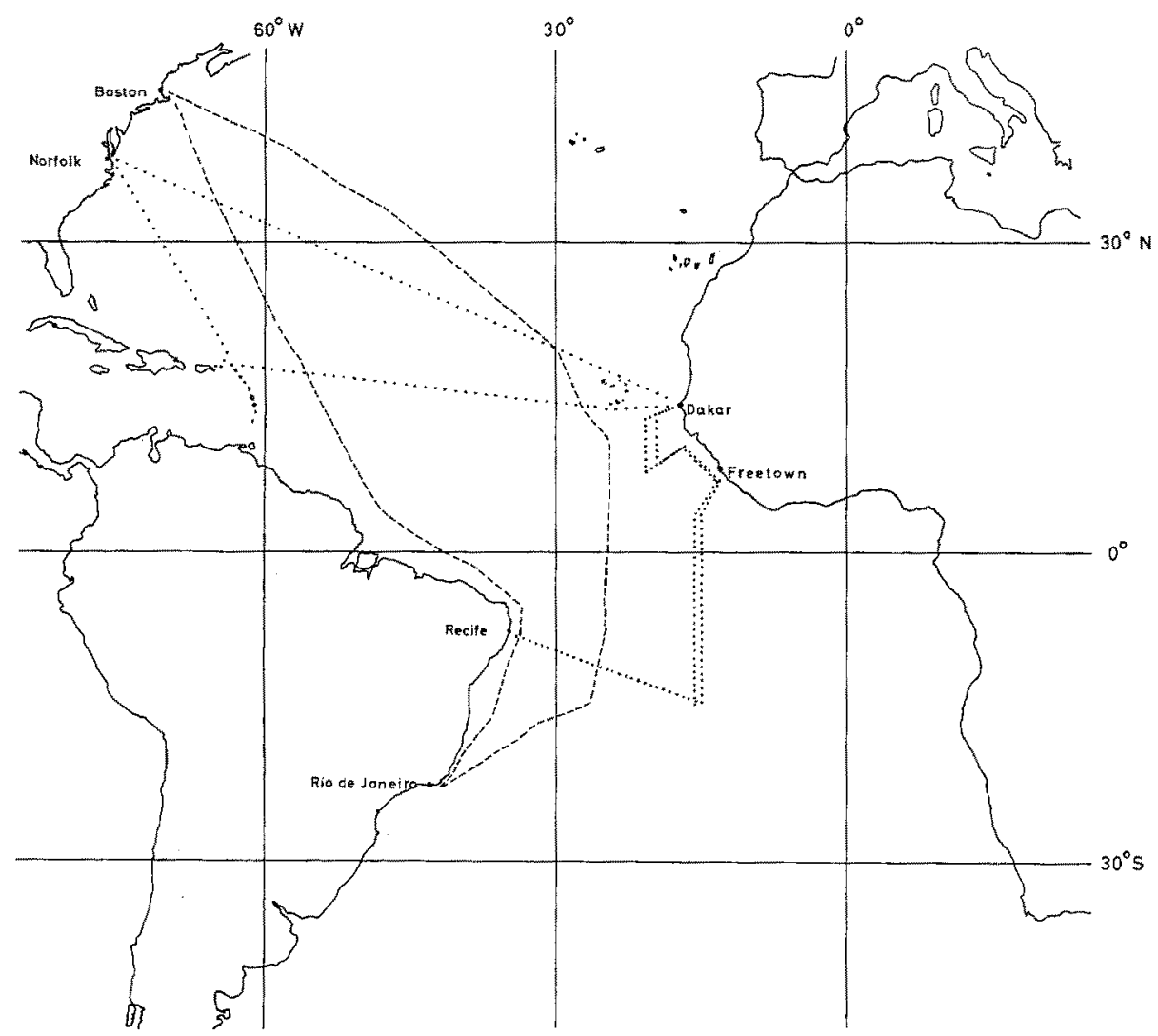

Abb. 2: Forschungsfahrten der USC+GSS "Explorer", 1. 2. bis 22. 4. 1963, punktiert, und der USCGC „Casco", 23. 7. bis 5. 9. 1963, gestrichelt

graphischen Station trieb. Das Netz war an einem Draht über einem schweren Gewicht so angebracht, daß es beim Fieren und Hieven fischte. Das Verdrängungsvolumen des erhaltenen Planktons wurde vom Bureau of Commercial Fisheries, Biological Laboratory, Washington D.C., bestimmt oder soll dort noch gewonnen werden. Für die Fahrt der "Explorer" sind die Ergebnisse schon veröffentlicht (National OcEanographic Data Center 1964). Für die Fänge der "Casco" sind mir nur die an Bord ermittelten Absetzvolumina bekannt. Uber das aus den Proben der „Explorer“ gefundene Verhältnis Verdrängungsvolumen/Absetzvolumen $\approx 1 / 4$ (ohne große Streuung) werden auch für die Fänge der "Casco" Angaben über das Verdrängungsvolumen gemacht. 
Zur Bestimmung von Mikroplankton-, Sestongehalt und Primärproduktion wurden Proben aus innen metallfreien Van-Dorn-Schöpfern benutzt. Das Seston wurde durch Vakuumfiltration von 2 bis 5 Liter Wasser auf gehärteten Papierfiltern (SCHLEICHER \& SchüLl Nr. 575, Porenweite etwa $1 \mu$ ) gewonnen. Das Gewicht der trockenen Filter wurde an Land vor und nach der Benutzung ermittelt; der Seston-Belag kann dabei auf $\pm 0,1 \mathrm{mg}$ genau gewogen werden. Danach wurden die Filter zur Eiweißbestimmung verwendet (KREY et al. 1957); die erhaltenen Albuminäquivalente können mit den von Hagmeier (1960) gegebenen Faktoren in Trocken- und Lebendgewicht (Biomasse) des Planktons umgerechnet werden. Ist die Zusammensetzung des abfiltrierten Planktons nicht bekannt, so erhält man nur Näherungswerte. Das im folgenden angegebene Plankton-Trockengewicht aus den Filterproben wäre bei Diatomeenplankton um $25 \%$ zu exhöhen, für Copepoden um $25 \%$ zu reduzieren. Das aus dem Eiweiß-Gehalt ermittelte Plankton-Lebendgewicht wäre für Diatomeen um $25 \%$ höher, für Peridineen um $25 \%$ geringer.

Zur Schätzung der Primärproduktion wurden zuerst mit einem Photometer die Tiefen von 50,25, 10 und $1 \%$ des an der Oberfläche einfallenden Lichtes gemessen. Proben von der Oberfläche und aus diesen 4 Tiefen wurden mit ${ }^{14} \mathrm{C}$ versetzt und in einen mit Oberflächenwasser gekühlten Inkubator gebracht, in dem sie unter natiirlichem Licht durch Graufilter etwa den gleichen Beleuchtungsverhältnissen wie an der Stelle der Entnahme unterworfen waren. Nach 24 Stunden wurden die Proben aus hellen und verdunkelten Flaschen filtriert, die Filter mit sehr schwacher Salzsäure gewaschen und getrocknet. Von der Auswertung durch das Bureau of Commercial Fisheries, Biological Laboratory, Washington, sind die Daten der "Explorer" schon veröffentlicht (National Oceanographic Data Center 1964). Die hydrographischen Daten wurden mir freundlicherweise nach der Korrektur schon an Bord der „Explorer“ und „Casco“ zur Verfügung gestellt.

\section{DIE VERTIKALVERTEILUNG VON SESTON UND EIWEISS}

\section{Tiefen-Mittelwerte aus dem tropischen Atlantik}

Die für den Seston- und Eiweißgehalt benachbarter Proben ermittelten Werte waren sehr häufig unterschiedlich. Bei niedrigen absoluten Werten sind große relative Abweichungen methodisch bedingt. Zum Ausgleich und zur Übersicht wurden Sestonund Eiweißwerte zunächst für verschiedene Tiefenhorizonte zusammengefaßt. Eine statistische Prüfung, wie weit die gefundenen Unterschiede bedeutsam sind, wurde noch nicht angestellt. Sicher sind mit den Daten aus mehreren Wasserkörpern auch Proben aus verschiedenen Populationen zusammengefaßt, die für eine genauere Untersuchung getrennt werden müßten. $\mathrm{Zu}$ den in ähnlicher Weise gewonnenen. Mittelwerten aus dem Geophysikalischen Jahr ergeben sich indes deutliche Differenzen.

Doch zunächst zu den Mittelwerten aus den Proben der "Explorer" und "Casco" selbst, die in den Tabellen 1 und 2 aufgeführt sind. Die Daten der „Explorer" wurden in drei Gruppen zusammengefaßt. Auf den Stationen der Schnitte vor der Küste sind im Mittel Eiweiß- und Sestongehalt höher als in der Nähe und südlich des Äquators. Einzelne hohe Werte wurden aber auch im offenen Ozean gefunden, wie niedrige in 
Tabelle 1

Mittelwerte von Seston und Eiweiß (Albumin-Aquivalente) für die biologischen Stationen der "Explorer", EQUALANT I

a) $4^{0}-15^{\circ} \mathrm{N}, 12^{\circ} 30^{\circ} \mathrm{W}$ (Stationen vor der afrikanischen Küste); b) $4^{0} \mathrm{~N}-15^{\circ} \mathrm{S}, 15^{0}-15^{\circ} 30^{\prime} \mathrm{W}$ (Schnitte senkrecht zum Aquator); c) $8^{0} 30^{\prime}-15^{\circ} \mathrm{S}, 15^{\circ}-34^{\circ} \mathrm{W}$ (Oberflächenproben); n: Zahl der Einzelwerte (in Klammern)

\begin{tabular}{|c|c|c|c|c|c|c|}
\hline $\begin{array}{l}\text { Schicht } \\
\text { (m) }\end{array}$ & $\begin{array}{l}\text { Mittlere } \\
\text { Tiefe } \\
\text { der Proben } \\
\text { (m) }\end{array}$ & $\left(\mathrm{mg} / \mathrm{m}^{3}\right)$ & (n) & $\left(\mathrm{mg} / \mathrm{m}^{3}\right)$ & (n) & $\begin{array}{c}\text { Plankton- } \\
\text { anteil } \\
\text { am Seston } \\
(\% / 0)\end{array}$ \\
\hline \multicolumn{7}{|c|}{ a) Stationen 5-29, 94-125 } \\
\hline 0 & 0 & 250 & (19) & 28 & (18) & 45 \\
\hline $1-10$ & 7 & 110 & (8) & 22 & (10) & 80 \\
\hline $11-30$ & 22 & 150 & (22) & 17 & (23) & 45 \\
\hline $31-50$ & 43 & 140 & (26) & 15 & (27) & 45 \\
\hline $51-75$ & 74 & 130 & (13) & 9 & (16) & 30 \\
\hline $76-100$ & 97 & 80 & (14) & 6 & (15) & 30 \\
\hline $101-150$ & 139 & 90 & (13) & 10 & (13) & 45 \\
\hline $151-300$ & 258 & 100 & (11) & 6 & (11) & 25 \\
\hline $301-500$ & 439 & 110 & (10) & 7 & (10) & 25 \\
\hline $501-1000$ & 890 & 70 & (6) & 8 & (6) & 45 \\
\hline $1001-2000$ & 1840 & 110 & (7) & 10 & (7) & 35 \\
\hline \multicolumn{7}{|c|}{ b) Stationen 33-90 } \\
\hline & 0 & 170 & (15) & 11 & (14) & 25 \\
\hline $1-10$ & 7 & 30 & (1) & 0 & (1) & - \\
\hline $11-30$ & 23 & 80 & (19) & 4 & (18) & 20 \\
\hline $31-50$ & 46 & 100 & (19) & 5 & (18) & 20 \\
\hline $51-75$ & 74 & 100 & (14) & 3 & (14) & 10 \\
\hline $76-100$ & 96 & 100 & (15) & 3 & (13) & 10 \\
\hline $101-150$ & 138 & 100 & (14) & 2 & (14) & 10 \\
\hline $151-300$ & 288 & 100 & (14) & 5 & (14) & 20 \\
\hline $301-500$ & 472 & 70 & (12) & 4 & (11) & 25 \\
\hline $501-1000$ & 924 & 50 & (12) & 3 & (11) & 25 \\
\hline $1001-2000$ & 1895 & 60 & (12) & 4 & (11) & 25 \\
\hline \multicolumn{7}{|c|}{ c) Station 59-Recife } \\
\hline 0 & 0 & 90 & (21) & 8 & (21) & 35 \\
\hline
\end{tabular}

der Nähe der Küste. Angereicherte und ärmere Wasserkörper sind vielfach miteinander verwirbelt. Vor der Küste bringt der Kanarenstrom nährstoff- und planktonreiches Wasser aus dem Auftriebsgebiet nördlich von Dakar mit; der Kontakt mit dem Boden und die Querzirkulation des Guineastromes sorgen außerdem für eine erhöhte Produktivität vor der Küste. Auf dem Aquatorschnitt sind ebenfalls durch die Querzirkulation lokale Anreicherungen zu erwarten.

Bemerkenswert sind bei küstennahen und küstenfernen Stationen die Maxima von Seston und Eiweiß an der Oberfläche. Da die Oberflächenproben mit einem Plastikeimer so genommen wurden, daß das Schiff sie nicht hat verunreinigen können, muß eine echte Anreicherung an der Oberfläche beobachtet worden sein. Der Gehalt der Oberflächenproben ist im südlichen Atlantik am geringsten (Tab. 1c). Aus dem Eiweißgehalt kann man nun auf das Trockengewicht des Planktons schließen (S. 274). Danach wird in der letzten Spalte der Tabelle 1 der Planktonanteil am Seston geschätzt. Für ozeanische Verhältnisse ist er sehr gering. 
Demgegenuiber läßt sich in den Proben der "Casco" (Tab. 2) das Seston fast vollständig als lebendes Plankton erklären. Der absolute Sestongehalt ist in allen Tiefen deutlich geringer als in den Proben der „Explorer". Wieder finden sich die Maxima von Seston und Plankton an der Oberfläche.

Tabelle 2

Mittelwerte von Seston und Eiweiß (Albumin-Aquivalente) für die biologischen Stationen der "Casco “, EQUALANT II

a) $39^{\circ} \mathrm{N}-22^{\circ} \mathrm{S}, 25^{\circ}-59^{\circ} \mathrm{W}$; b) $23^{\circ} \mathrm{S}-38^{\circ} \mathrm{N}, 34^{\circ} 30^{\prime}-68^{\circ} \mathrm{W}$ (Oberflächenproben); $\mathrm{n}$ : Zahl der Einzelwerte (in Klammern)

\begin{tabular}{|c|c|c|c|c|c|c|}
\hline $\begin{array}{l}\text { Schicht } \\
\text { (m) }\end{array}$ & $\begin{array}{l}\text { Mittlere } \\
\text { Tiefe } \\
\text { der Proben } \\
\text { (m) }\end{array}$ & Seston & (n) & $\left(\mathrm{mg} / \mathrm{m}^{3}\right)$ & (n) & $\begin{array}{l}\text { Plankton- } \\
\text { anteil } \\
\text { am Seston } \\
(0 / 0)\end{array}$ \\
\hline \multicolumn{7}{|c|}{ a) Stationen $2-58$} \\
\hline 0 & 0 & 80 & (25) & 17 & (25) & 85 \\
\hline $1-10$ & 5 & 40 & (25) & 12 & (25) & 100 \\
\hline $11-20$ & 15 & 30 & (26) & 10 & (25) & 100 \\
\hline $21-30$ & 25 & 40 & (23) & 11 & (24) & 100 \\
\hline $31-40$ & 35 & 50 & (22) & 12 & (24) & 95 \\
\hline $41-50$ & 49 & 30 & (19) & 9 & (21) & 100 \\
\hline $51-75$ & 70 & 40 & (26) & 11 & (26) & 100 \\
\hline $76-100$ & 94 & 40 & (29) & 11 & (29) & 100 \\
\hline $101-150$ & 131 & 20 & (23) & 10 & (23) & 100 \\
\hline $151-300$ & 251 & 20 & (29) & 7 & (26) & 100 \\
\hline $301-600$ & 445 & 40 & (23) & 9 & $(22)$ & 90 \\
\hline \multicolumn{7}{|c|}{ b) Rio de Janeiro - Boston } \\
\hline 0 & 0 & 60 & $(113)$ & 16 & (112) & 100 \\
\hline
\end{tabular}

\section{Vergleiche mit Mittelwerten aus dem Nordatlantik und dem Indischen Ozean}

Für Vergleiche mit den im tropischen Atlantik gefundenen Mittelwerten stehen Daten aus dem Nordatlantik und dem Indischen Ozean zur Verfügung (KREY 1964, Hagmeier 1964). In Abbildung 3 sind die Mittelwerte aus den Fahrten im Internationalen Geophysikalischen Jahr der "Anton Dohrn " und der "Gauß" und von einer Reise der "Diamantina" neben die Ergebnisse von der "Explorer" und der "Casco" gestellt. Der Sestonwert wird jeweils von den beiden gebrochenen Linien eingeschlossen; durch die Schraffur ist der Teil gekennzeichnet, den wir als unbelebt ansehen können. Der belebte Teil links von der vertikalen Trennungslinie wurde aus dem Eiweißwert mit dem Faktor 4 berechnet - deshalb kann man auch den Eiweißgehalt in den verschiedenen Tiefen direkt ablesen. Der erhaltene Wert für das Plankton-Trockengewicht kann in einer an Diatomeen reichen Oberschicht zu gering ausfallen, in Tiefen mit vielen Zooplanktern zu hoch (vgl. KREY 1964, p. 26).

Die Proben der "Anton Dohrn“ sind durch einen besonders hohen Gehalt an unbelebtem Seston (Tripton) ausgezeichnet. $\mathrm{K}_{R E Y}(1964)$ vermutet hier aus abgeschmolzenem Landeis stammendes anorganisches Material, dessen Tiefenverteilung sich durch das Abschmelzen im Sommer und das Absinken im Winter deuten läßt. In gleichem 
Sinne ist der Gang des als Folge des Planktonbestandes entstehenden Detritus. Der sehr ausgeprägte Unterschied zwischen dem Planktongehalt in Sommer und Winter in höheren Breiten verringert sich in niederen. Schon in den Daten der "Gauß" ist er weit weniger ausgeprägt. Die Planktonmenge ist in dem von der "Gauß" untersuchten Gebiet noch relativ groß: die Nährstoffversorgung wird durch die Querzirkulation in den Asten des hier durchziehenden Nordatlantischen Stromes begünstigt.

Uber der Dichte-Sperrschicht in den Tropen ist dann der Planktongehalt sehr gering (Proben der "Casco") - als Folge gibt es auch nur wenig Detritus und Zooplankton in der Tiefe. Unter dem Einfluß von Querzirkulation und Auftriebswasser findet man auch in den Tropen reichere Gebiete (Proben der „Explorer“). Der vor der afrikanischen Küste beobachtete höhere Triptongehalt wird zum Teil aus feinen Teilchen bestehen, die von den Wüsten ins Meer hinausgeweht sind. Der hohe Triptonanteil auf dem Äquator-Schnitt der "Explorer" ist mir noch unerklärlich.

Die angeführten Daten von einer Fahrt der "Diamantina" zeigen die Verhältnisse in dem von starken Strömungen und Auftriebswasser angereicherten Gebiet $z$ wischen Australien und Indonesien. Im zentralen Indischen Ozean werden der Sestonund Planktongehalt geringer sein.

\section{Maxima in der Tiefenverteilung von Seston und Eiweiß}

Die Ansammlung von Seston und Plankton an der Oberfläche wurde schon bei der Darstellung der mittleren Vertikalverteilung deutlich. Eine Anreicherung an oder in der Dichtesprungschicht, so wie sie von KREY (1954) in der Nordsee oder von SorokIN (1960) für den tropischen Atlantik beschrieben wurden, konnte bei den Tiefen-Mittelwerten wegen der unterschiedlichen Tiefe der Sprungschicht nicht auffallen. Betrachtet man jede der Stationen mit einem deutlichen Dichtesprung für sich, so findet man bei $80 \%$ das schon erwähnte Maximum an der Oberfläche; ein meist sekundäres Maximum von Seston erscheint an 29, von Eiweiß an 22 der untersuchten 53 Stationen in der Sprungschicht, gewöhnlich in ihrem mittleren oder unteren Abschnitt. Dazu muß aber bemerkt werden, daß die Probennahme nicht auf diese Ansammlungen geridhtet war. Nach den Erfahrungen aus der Nordsee sind die Maxima oft von so geringer Tiefenausdehnung, daß es selbst nach der Ortung durch einen Trübungsmesser schwer ist, eine Wasserprobe aus der interessierenden Konzentration zu erhalten. Auf den Stationen der "Explorer" und "Casco" können deshalb in vielen Fällen die Anreicherungen in der Sprungschicht nicht erfaßt sein. Ein Eindruck von ihrer Beständigkeit und Ausdehnung läßt sich vielleicht noch aus den Echogrammen erkennen. Eine genauere Studie über ihren Umfang und die Bedeutung der Sprungschicht für eine Remineralisation etwa müßte jedoch Trübungsmessungen und eine größere Probenzahl enthalten.

Leider reicht auch das Material für eine genauere Untersuchung über die Auswirkungen des äquatorialen. Unterstromes auf die Verteilung von Seston und Plankton nicht aus. Außergewöhnliche Werte wurden in seinem Kern (der hydrographisch durch Wasser von über 36,2\% Salzgehalt gut definiert ist) oder in dessen Umgebung nicht beobachtet. Seine Energie sollte aber die Nährstoffversorgung der Dedschicht am Aquator noch verbessern. Die als deren Folge gefundenen Plankton- und Sestonmaxima werden im nächsten Kapitel behandelt. 


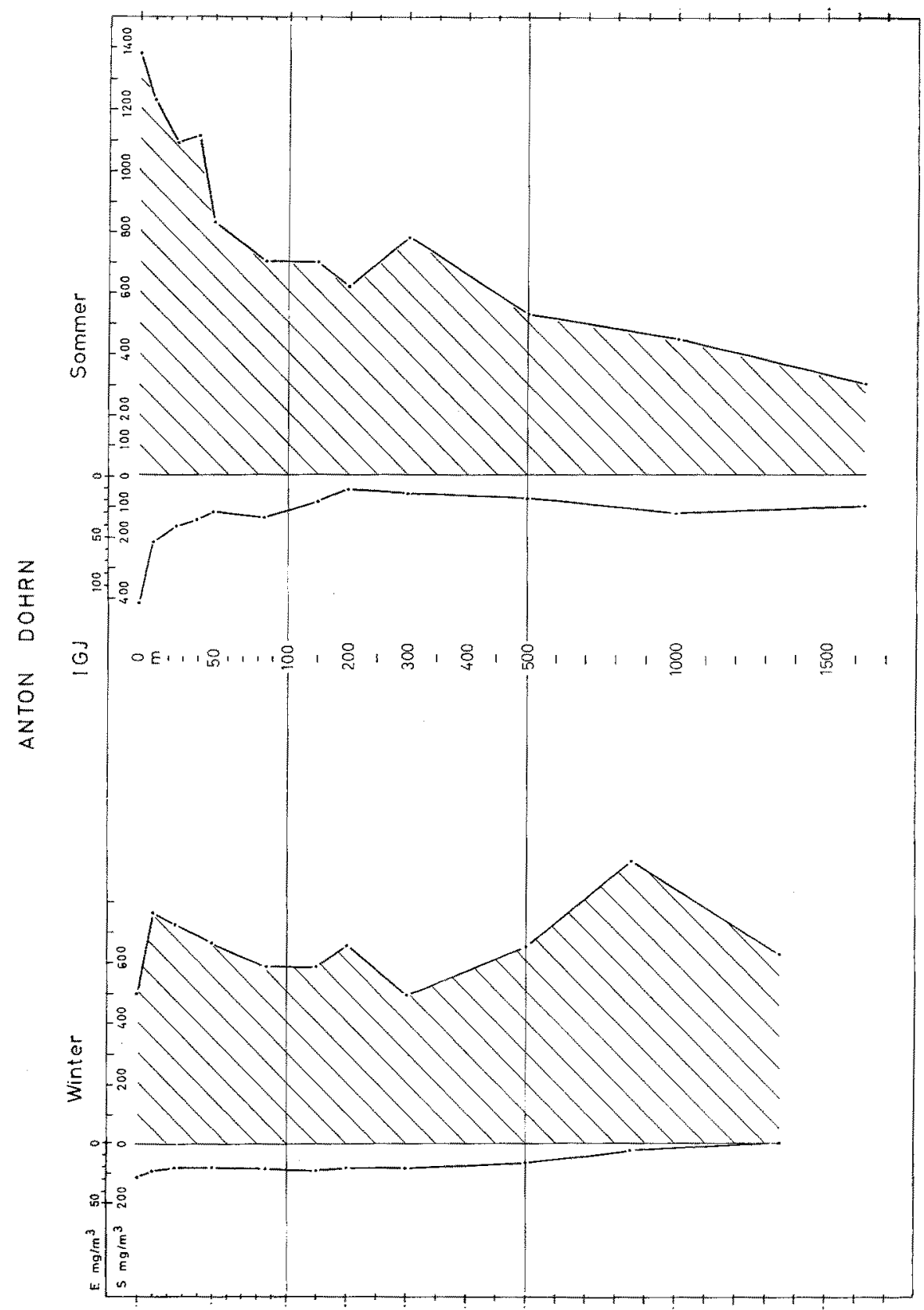

Abb. 3A: Tiefen-Mittelwerte für Seston und Eiweiß. „Anton Dohrn“, Nordatlantik, Winter: 6. 3. bis 16. 4. $1958,55^{0}-67^{\circ} \mathrm{N}, 20^{0}-45^{\circ} \mathrm{W}$; Sommer: 10.8 . bis $19.9 .1958,56^{\circ}-67^{\circ} \mathrm{N}$, $15^{\circ}-44^{0} \mathrm{~W}$ 


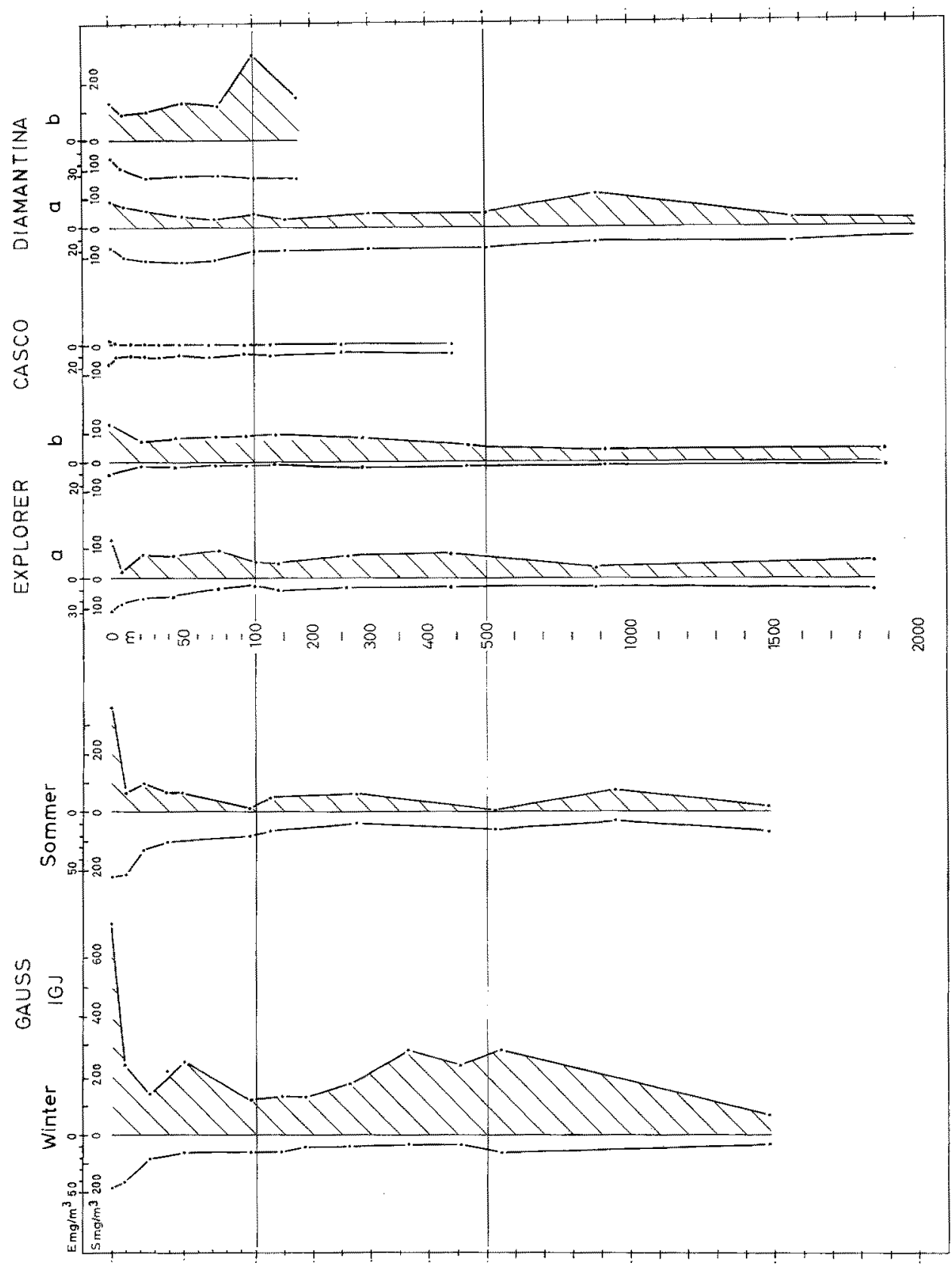

Abb. 3B: Tiefen-Mittelwerte für Seston und Eiweiß. "Gau ${ }^{*}$, Nordatlantik, Winter: 15. 3. bis 30. 4. $1958,39^{\circ}-55^{\circ} \mathrm{N}, 25^{\circ}-48^{\circ} \mathrm{W}$; Sommer: 5. 8. bis $21.9 .1958,42^{\circ}-56^{\circ} \mathrm{N}, 15^{\circ}-50^{\circ} \mathrm{W}$. "Explorer", tropischer Atlantik, a: Stationen im Guineastrom, 17. bis 24. 2., 29. 3. bis 9. 4. $1963,4^{0}-15^{\circ} \mathrm{N}, 12^{\circ} 30^{\prime}-21^{\circ} \mathrm{W}$; $b$ : Stationen in den Aquatorialströmen, 25. 2. bis 28. 3. 1963, $4^{\circ} \mathrm{N}-15^{\circ} \mathrm{S}, 15^{\circ}-15^{\circ} 30^{\prime} \mathrm{W}$. "Cascos, tropischer Atlantik: 25. 7. bis 4. 9. $1963,39^{\circ} \mathrm{N}-22^{\circ} \mathrm{S}$,

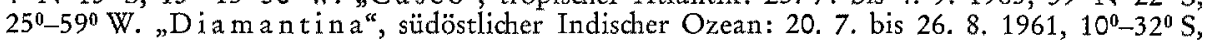
$112^{\circ}-130^{\circ} \mathrm{E}$; $a$ : ozeanische Stationen, $b$ : Schelfstationen. Seston (S): belebter Teil (Plankton) offen, unbelebter Teil (Tripton) schraffert. Aus dem nicht schraffierten Teil ist zugleich der Eiweißgehalt (E) ablesbar 


\section{PLANKTON UND SESTON IN DER DECKSCHICHT}

\section{Die Netzplankton-Biomasse}

Die Plankton-Netzfänge wurden gewöhnlich nachts ausgeführt, und zwar so tief, wie die gleichmäßig erwärmte Deckschicht reichte. Die Tiefe der durchfischten Schicht lag dementsprechend zwischen 25 und $180 \mathrm{~m}$; an der Küste wurde einige Male, wenn die Temperatur schon von der Oberfläche an gleichmäßig abfiel, der Netzfang bis in Bodennähe ausgedehnt. Abbildung $4 a$ zeigt die Lage der bearbeiteten Stationen. Für die "Explorer" stammt die angegebene Biomasse aus dem Verdrängungsvolumen der Plankter; für die "Casco" wurden die Daten, weniger sicher, aus dem Absetzvolumen berechnet (S. 273). Zeitlich sind in Abbildung 4 drei verschiedene Perioden zusammengebracht. Deshalb wurde nicht versucht, durch Isolinien Gebiete ähnlichen Gehalts zu verbinden.

Die höchsten Werte fanden sich zwischen der afrikanischen Küste und den Kapverdischen Inseln. Hier bringt der Kanarenstrom wechselnde Mengen planktonreiches Wasser aus dem Auftriebsgebiet nördlich von Dakar. Auch an der Küste zwischen Dakar und Freetown ist das Netzplankton reichhaltiger als im offenen Ozean. Nach dem Schema der Abbildung $1 b$ können wir am Aquator wieder Auftriebswasser erwarten und damit in Aquatornähe gefundene Zooplanktonmaxima erklären. Aber auch weiter südlich liegen noch einige "Explorer"-Stationen mit reichen Planktonfängen. Eindeutiger als auf dem Aquator-Schnitt der „Explorer" kommt der Wechsel $\mathrm{z}$ wischen der verarmten Deckschicht und reichen Planktonvorkommen in Divergenzgebieten bei den Stationen der "Casco" zum Ausdruck. Auf niedrige Werte in der Sargasso-See folgt eine Erhöhung an der Grenze von Nordäquatorial- und Gegenstrom (vgl. Abb. 1); nach einem Absinken des Planktongehalts auf dem weiteren Wege nach Süden erfolgt ein erneuter Anstieg am Äquator. Die niedrigsten Werte wurden schließlich im südlichen Atlantik gefunden.

\section{Die Mikroplankton-Biomasse}

Zum Vergleich mit dem Netzplankton wurde für die gleiche Schicht aus den gemessenen Albumin-Äquivalenten der Mikroplankton-Gehalt bestimmt (S. 274). Die in Abbildung $4 \mathrm{~b}$ gegebenen Daten sind nach Armstrong (1954) als "IMK-Werte“ berechnet.

Die Beziehung zu den. Divergenzgebieten ist weniger deutlich als beim Netzplankton. Der Einfluß des Auftriebwassers vor der afrikanischen Küste macht sich dagegen sehr wohl bemerkbar. Neben dem Maximum findet sich hier allerdings auch das Minimum der beobachteten Bestände. Uberhaupt besteht zwischen den etwa

Abb. 4: $a$ : Biomasse des Netzplanktons, $b$ : des Mikroplanktons, $\mathrm{mg} / \mathrm{m}^{2}$, oberhalb der Temperatur-Sprungschicht. Ostlich $21^{\circ} \mathrm{W}$ : aus Fängen und Schöpfproben der "Explorer ${ }^{\text {. }}$. Gerade Zahlen, offene Stationskreise: 16.2 . bis 7.3 . 1963; kursive Zahlen, ausgefullte Kreise: 20.3. bis 9. 4. 1963. Westlich 240 W: aus Proben der "Casco", 1. bis 19. 8. 1963. Die endgültigen Werte aus den Netzfängen sind noch nicht bekannt; angegebene Zahlen deshalb in Klammern 

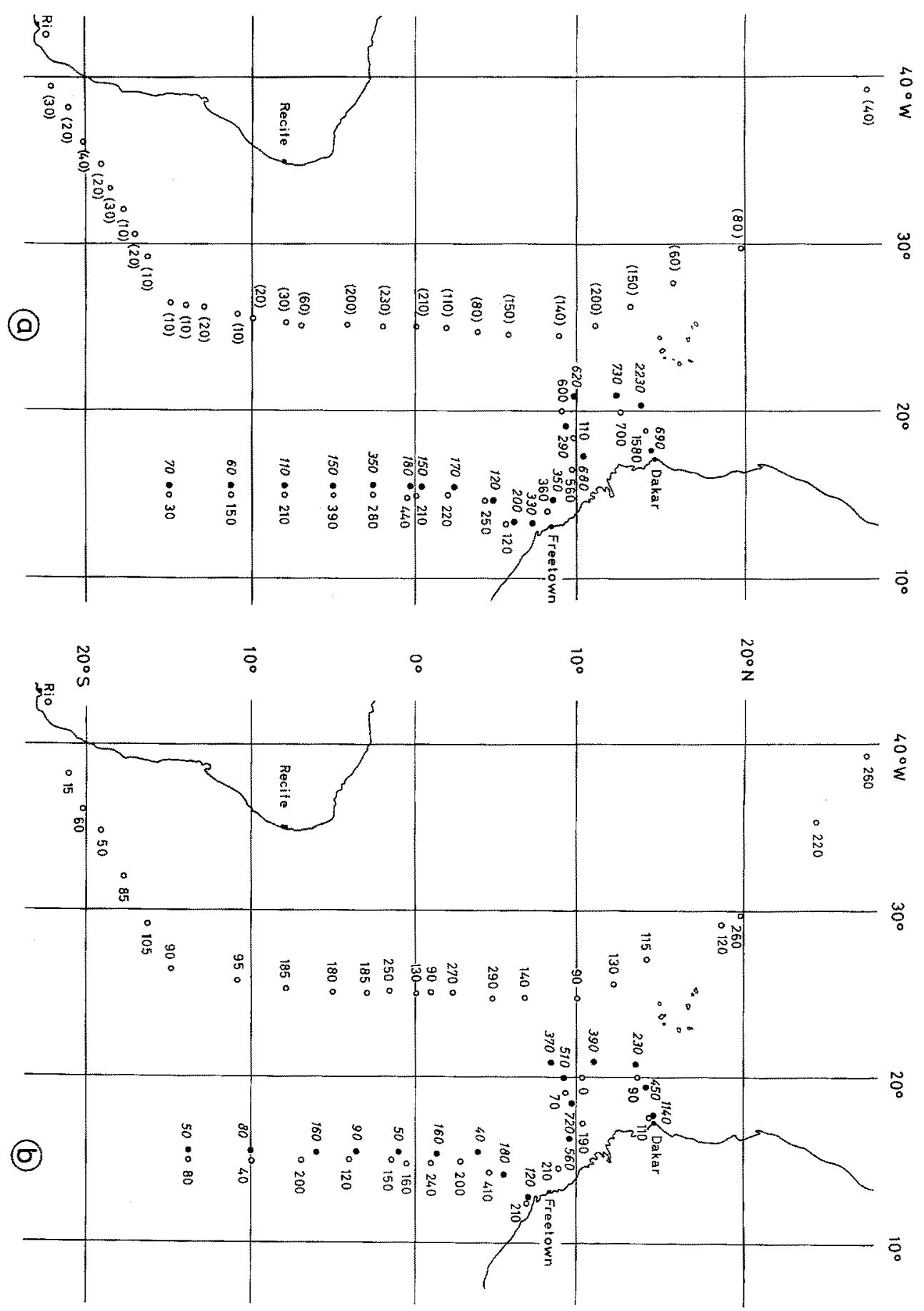
6 Wochen auseinanderliegenden ersten und letzten Stationen der "Explorer" ein großer Unterschied im Planktongehalt. Das kann die Folge einer verschieden starken Beimengung von Auftriebswasser sein - die Oberflächen-Temperatur weist allerdings nicht darauf hin. Andererseits wurden im Divergenzgebiet unter dem Aquator bei den Schnitten der "Explorer" und "Casco" eine deutliche Temperaturdepression und ein Anstieg des Phosphatgehaltes an der Oberfläche beobachtet, ohne daß der Mikroplanktonbestand dazu eine Beziehung gezeigt hätte. Viele Unregelmäßigkeiten können auch durch eine verschieden starke Zehrung durch das Zooplankton gedeutet werden.

\section{Das Biomassen-Verbältnis Netzplankton zu Mikroplankton}

Nimmt man einmal an, daß die Masse des Netzplanktons vornehmlich aus Konsumenten, die des Mikroplanktons hauptsächlich aus (Primär-)Produzenten besteht, so erscheint eine Untersuchung über das Verhältnis zwischen diesen beiden Gruppen besonders reizvoll. Netz- und Mikroplanktonstationen wechseln meistens einander ab; für einzelne Stationen lassen sich deshalb keine genauen Verhältniszahlen, sondern nur interpolierte Werte angeben. Als Mittelwerte für das Verhältnis Netzplankton zu Mikroplankton ergeben sich aus den Proben der "Explorer" 1:0,7, aus den. Proben der "Casco" 1:3,1. Die Ernährungsbasis für die Konsumenten wäre also nur klein. Aus Vergleichsfängen im südöstlichen Indischen Ozean ließ sich ein Verhältnis von 1:8 angeben (HAGmeier 1964), was etwa den Vorstellungen über die Bestände in der Nahrungskette in höheren Breiten entspricht. Ahnliche Verhältnisse finden sich nun auch auf dem Schnitt der "Casco" im offenen Ozean nördlich und südlich des Aquators. In Divergenzgebieten am Aquator und in $10^{\circ} \mathrm{N}$ war dagegen die MikroplanktonBiomasse meistens kleiner als die Biomasse des Netzplanktons, ebenso in dem verhältnismäßig gut mit Nährstoffen versorgten Wasser vor der afrikanischen Küste. Gerade hier sollte sich aber ein reduzierter Mikroplanktonbestand schnell wieder erholen können.

Nach dieser Bestandsaufnahme erscheint es nötig und interessant, Nahrungsbedarf und Vermehrungsgeschwindigkeit genauer zu untersuchen und eine Vorstellung darüber zu erhalten, bis zu welcher Verdünnung ein Mikroplanktonbestand von den Konsumenten abgefischt werden kann. Zu einer gründlichen Auswertung der EQUALANTDaten fehlen leider Proben, aus denen man durch Zählungen die Zusammensetzung des Mikroplanktons erfahren kann. Die vorher erwähnten Unregelmäßigkeiten im Mikroplanktonbestand erscheinen aber auch ohne genaue Analyse durch die Einwirkungen verhältnismäßig großer Zooplanktonbevölkerungen verständlich.

\section{Das Seston in der Deckschicht}

Abbildung 5 zeigt die mittleren Sestongehalte der warmen Deckschicht; sie reichen von $10 \mathrm{bis} 1280 \mathrm{mg} / \mathrm{m}^{3}$. Das Minimum ist in einer 5-Liter-Wasserprobe kaum meßbar, das Maximum ist für die Gewässer um Helgoland ein normaler Wert. Deutlich unterscheiden sich, wie schon bei der Darstellung der Tiefen-Mittelwerte, die Stationen der 


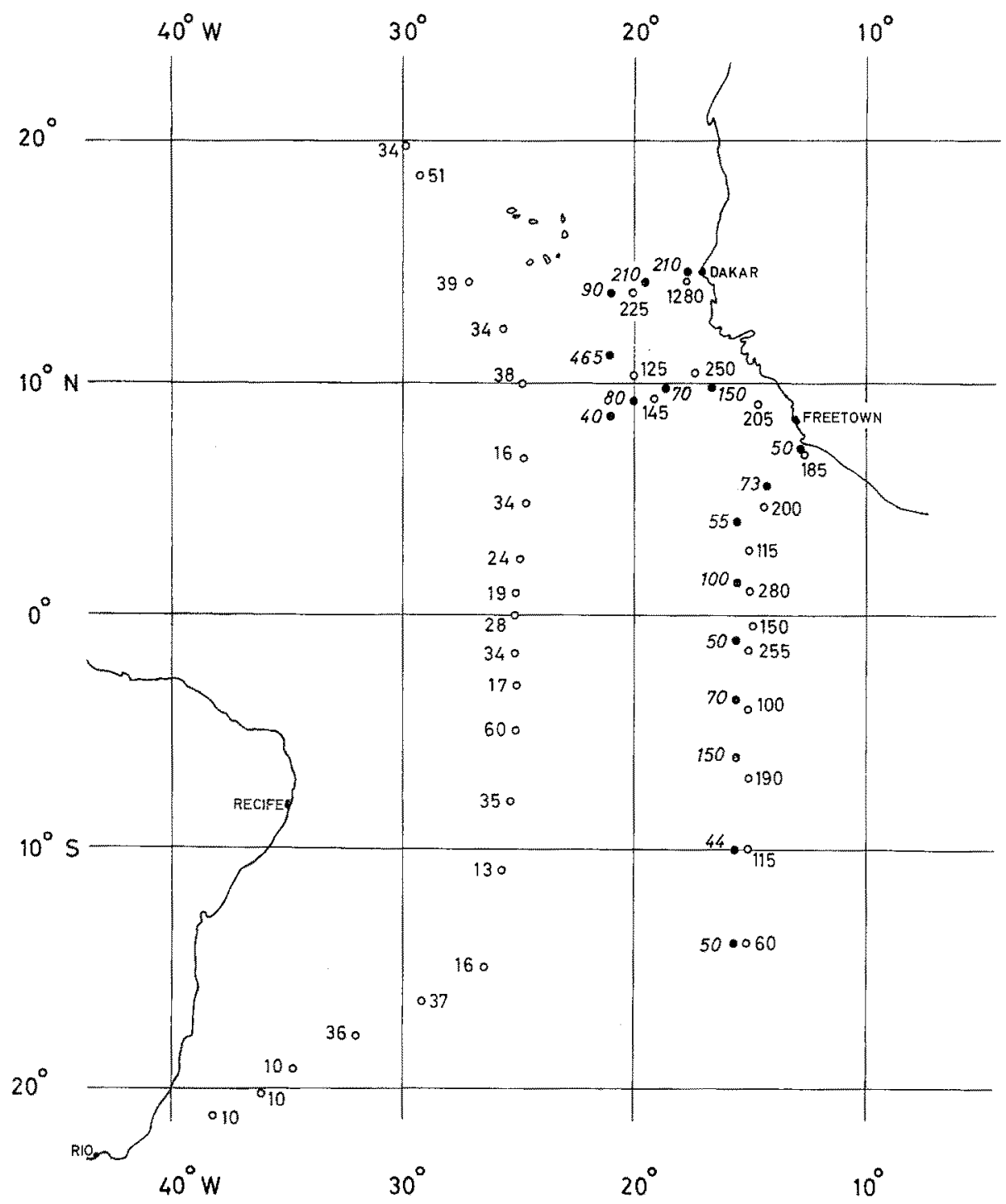

Abb. 5: Seston ( $\mathrm{mg} / \mathrm{m}^{3}$ ) oberhalb der Temperatur-Sprungschicht. Ostlich $21^{\circ} \mathrm{W}$ : Stationen der "Explorer". Gerade Zahlen, offene Kreise: 16. 2. bis 7. 3. 1963; kursive Zahlen, ausgefullte Kreise: 20. 3. bis 9. 4. 1963. Westlich 240 W: Stationen der "Casco", 1. bis 19.8. 1963

"Casco" von denen der "Explorer": neben der Differenz in den absoluten Sestonwerten auch durch den unerschiedlichen Planktonanteil, der für die Proben der "Casco“ nahezu $100 \%$ beträgt, für die Proben der "Explorer" im Mittel aber nur $28 \%$. Ansammlungen von Partikeln in Divergenzgebieten, wie sie in der Aquatorialregion des Pazifik von der "Albatross"-Expedition beobachtet wurden, machen sich in den Proben der "Explorer" und "Casco" nicht so deutlich bemerkbar. 
Die durch die "Explorer" in der ersten Untersuchungsperiode gefundenen Werte waren bei der Wiederholung der Schnitte im gleichen Gebiet oft sehr verändert. Station 1 mit dem Maxinum von $1280 \mathrm{mg}$ Seston dürfte ein Beispiel sein für die schon erwähnte Wirkung von Staubteilchen aus der Wüste: der Sestongehalt nahm von der Oberfläche (2500 mg, darin $2 \%$ Plankton) bis zu $150 \mathrm{~m}$ Tiefe $(310 \mathrm{mg}, 20 \%$ Plankton) gleichmäßig ab, wobei am Tage der Untersuchung und auch schon an den Vortagen östliche Winde und ein starker Dunst beobachtet wurden. Auch an den folgenden von der „Explorer" untersuchten Stationen blieb bei relativ hohen Sestonwerten der Planktongehalt gering - auf der Rückreise war dagegen hier der Planktonanteil wesentlich höher. Die laufenden Untersuchungen im Laboratoire d'Oceanographie de Tiaroye/Mer bei Dakar (Dr. Rossignol) sollen zu einem Verständnis der Herkunft der Wasserkörper in diesem Gebiet, ihrer Vermischung und ihres Einflusses auf die Entwicklung des Planktons beitragen.

\section{PRIMARPRODUKTION}

Die Ergebnisse der Versuche mit radioaktivem Kohlenstoff auf der „Explorer“ sind in Abbildung 6 a eingetragen. Danach bewegt sich die Primärproduktion zwischen 3,02 und $0,03 \mathrm{~g} \mathrm{C} / \mathrm{m}^{2}$ am Tag. In diesem Bereich liegen zugleich auch die meisten der bisher vom Meer bekanntgewordenen Produktionszahlen, wobei die Maxima in Auftriebsgebieten, die Minima in der vom Tiefenwasser abgeschlossenen tropischen Deckschicht gefunden wurden.

Auffällig sind bei den Stationen der „Explorer" wieder die großen Unterschiede zwischen zum Teil benachbarten Proben. Die Differenzen zwischen erster und zweiter Beobachtungsperiode vor der afrikanischen Küste werden zum großen Teil durch unterschiedliche Bestände von Phytoplanktern zu erklären sein. Einige wesentliche Schwankungen wird aber auch die Methodik verursacht haben. Die Proben waren ja dem natürlichen Licht ausgesetzt, dessen Intensität mit der Bewölkung sehr unterschiedlich ausfiel. So wurden auf der "Casco" mittags Beleuchtungsstärken zwischen 300 (im Regenschauer) und 40000 Lux gemessen (bei bedecktem Himmel im Mittel 9000 Lux). Ein relativ hoher Produktionswert liegt in der Nähe des Aquators, wo auch ein etwas erhöhter Phosphatgehalt in der Deckschicht gemessen wurde - zugleich liegen in dieser Zone aber auch niedrige Werte. Auf einer Dauerstation der "Lomonossow" wurde an aufeinanderfolgenden Tagen am Aquator eine Produktion von 400 und $151 \mathrm{mg} \mathrm{C} / \mathrm{m}^{2}$ bestimmt (National Oceanographic Data Center 1964); das Maximum wurde von der "Lomonossow" während der EQUALANT-I-Beobachtungen im Auftriebsgebiet nördlich von Dakar zu 4,09 $\mathrm{g} \mathrm{C} / \mathrm{m}^{2} / \mathrm{Tag}$ gefunden.

Eine Beziehung der Produktionsdaten auf den Phytoplanktonbestand ist leider nicht möglich; auch hier fehlen Proben, in denen das Mikroplankton gezählt werden könnte. Mit der Eiweißbestimmung wird ja nur die Summe der Phyto- und Zooplankter erfaßt. Immerhin erhält man damit den oberen Grenzwert für den photosynthetisch aktiven Planktonbestand. In Abbildung 6b ist dieser Bestand für die Schicht eingetragen, in der die Primärproduktion möglich erscheint (bis zu der Tiefe, in der noch $1 \%$ des an der Oberfläche einfallenden Lichtes gemessen wurde und die an der Küste 30 bis $50 \mathrm{~m}$, im offenen Ozean 55 bis $120 \mathrm{~m}$ betrug). Die gefundene Produktion 

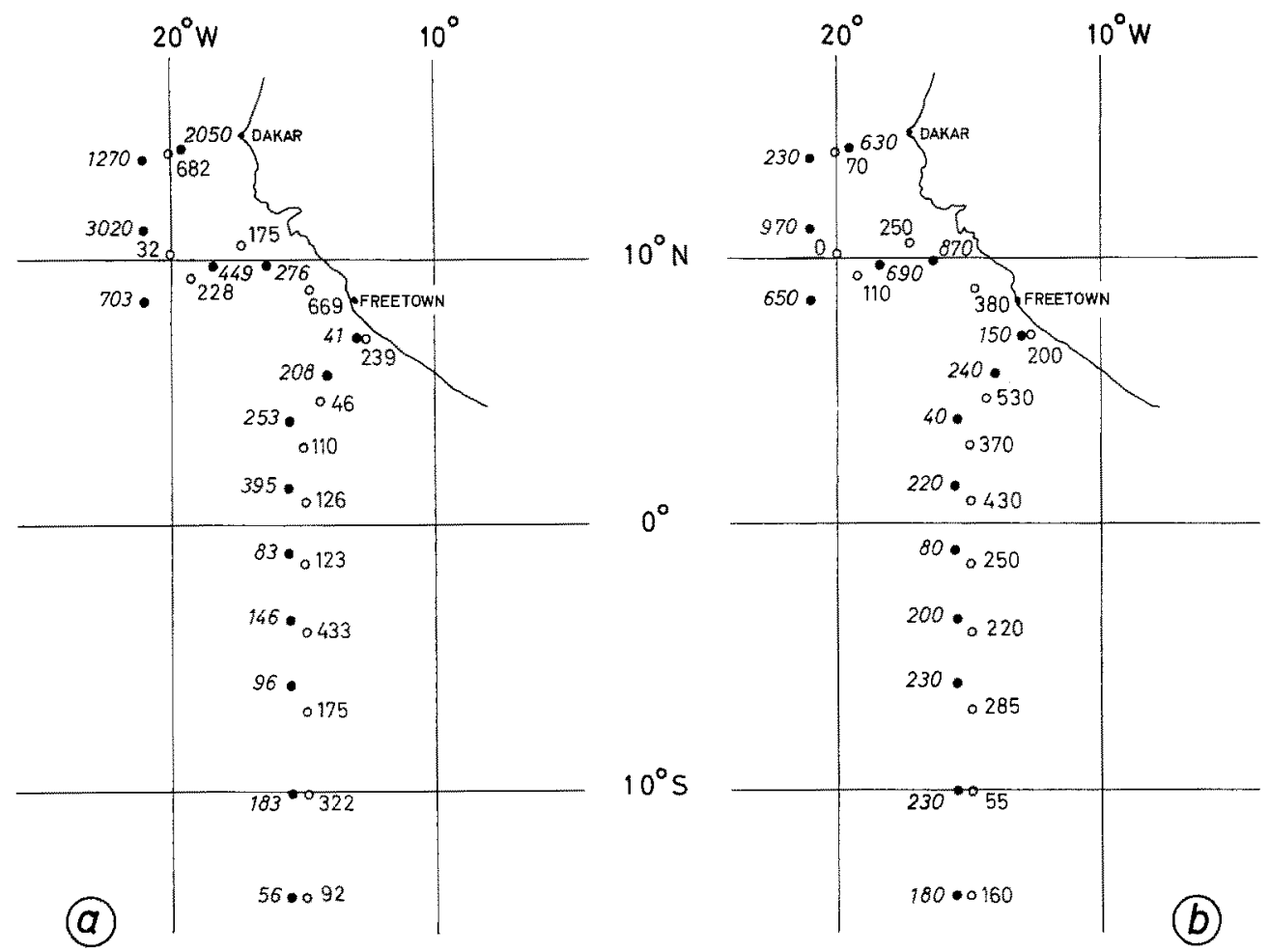

Abb. 6: a: Primärproduktion (mg C/m² $/ \mathrm{m}^{2} / \mathrm{Tg}$ ), $b$ : Mikroplankton-Bestand (mg C/m²) in der produktiven Deckschicht. Proben der "Explorer"; gerade Zahlen, offene Kreise: 16. 2. bis 6. 3. 1963 , kursive Zahlen, ausgefullte Kreise: 20.3 . bis 9.4 .1963

erreicht bei den Werten über $1,0 \mathrm{~g} \mathrm{C} / \mathrm{m}^{2}$ das Dreifache dieses Mikroplanktonbestandes, in einigen anderen Fällen das Doppelte, zweimal, wo der Planktonbestand vielleicht $\mathrm{zu}$ gering angegeben ist, das Sechsfache.

\section{ZUSAMMENFASSUNG}

1. Die hier vorgelegte Ausarbeitung enthält nur einen sehr kleinen Teil der Daten, die während der internationalen Untersuchungen seit $1963 \mathrm{im}$ tropischen Atlantik gewonnen wurden. Sie bringt erste Ergebnisse von zwei Reisen des Verfassers im Frühjahr und Sommer 1963.

2. Die gefundenen Werte von Seston und Eiweiß (Albumin-Äquivalente) waren im einzelnen sehr unterschiedlich; eine Deutung mit Hilfe von hydrographischen Daten genügte nicht in allen Fällen. Die Mittelwerte sind deutlich niedriger als die entsprechenden Zahlen aus dem Nordatlantik. Die höchsten Werte fanden sich an der Oberfläche; eine zweite Anreicherung wurde häufig in der Dichte-Sprungschicht beobachtet. Der aus den Eiweißzahlen errechnete Planktongehalt des Sestons war im östlichen Teil des untersuchten Gebietes auch im offenen Ozean unerwartet gering. 
3. Die Biomasse des Netzplanktons übertraf in gut mit Nährstoffen versorgtem Wasser die Biomasse des Mikroplanktons. Nur durch eine hohe Primärproduktion, wie sie in diesen Gebieten auch vielfach gemessen wurde, können die beobachteten Planktonbestände erhalten werden.

4. Die gefundenen Verhältnisse lassen umfassendere Beobachtungen in tropischen Gebieten und Versuche iber Nahrungsbedarf und Vermehrung der Planktonorganismen wünschenswert erscheinen.

Die Möglichkeit zur Teilnahme am ICITA-Programm verdanke ich der Initiative und Unterstützung von Herrn Prof. Dr. J. KREY (Institut für Meereskunde der Universität Kiel) und den Geldmitteln, die durch das Bundesministerium für Ernährung, Landwirtschaft und Forsten bereitgestellt und durch die Deutsche Förderungsgesellschaft für Entwicklungsländer zu meiner Betreuung verwendet wurden. Während der Untersuchungsperiode EQUALANT I war ich Gast auf dem United States Coast and Geodetic Ship „Explorer", während EQUALANT II auf dem United States Coast Guard Cutter "Casco“. Für die freundliche Aufnahme an Bord und die tatkräftige Unterstützung bin ich den Herren Kommandanten, Captain John C. Butl der "Explorer" und Commander Paul A. LuTz der "Casco", ihren Offizieren und Mannschaften zu herzlichem Dank verpflichtet. Herrn Prof. Dr. G. Neumann und Herrn Prof. Dr. W. PIERson, New York University, danke ich für die harmonische Zusammenarbeit. - Für ihre sorgfältige Hilfe bei der Aufarbeitung yon Seston- und Eiweißproben und bei der Darstellung der Ergebnisse bin ich Frl. P. Herford und Frl. U. Junghans, Kiel, sowie Frl. K. LANGE und Frl. H. BAY, Helgoland, sehr dankbar.

\section{ZITIERTE LITERATUR}

Armstrong, F. A. J., 1954. Phosphorus and silicon in sea water off Plymouth during the years 1950 to 1953. J. Mar. biol. Ass. U. K. 33, 381-392.

CREASE, J. \& POGSON, A., 1964. Observations of the equatorial undercurrent by submarine. Deep Sea Res. 11, 391-394.

Hagmeier, E., 1961. Plankton-Äquivalente. Kieler Meeresforsch. 17, 32-47.

- 1964. Zum Gehalt an Seston und Plankton im Indischen Ozean zwischen Australien und Indonesien. Kieler Meeresforsch. 20, 12-17.

KreY, J., 1954. Beziehungen zwischen Phytoplankton, Temperatursprungschicht und Trübungsschirm in der Nordsee im August 1952. Kieler Meeresforsch. 10, 3-19.

- 1964. Die mittlere Tiefenverteilung von Seston, Mikrobiomasse und Detritus im nördlichen Nordatlantik. Kieler Meeresforsch. 20, 18-29.

- Banse, K. \& Hagmerer, E., 1957. Über die Bestimmung von Eiweiß im Plankton mittels der Biuretreaktion. Kieler Meeresforsch. 13, 35-40.

Metcal., W. G., Voorhis, A. D. \& Stalcup, M. C., 1962. The Atlantic equatorial undercurrent. J. geophys. Res. 67, 2499-2508.

National Oceanographic Data Center, 1964. Data report; Equalant I. Washington, D. C.

Sснотт, G., 1943. Nachtrag zu dem Aufsatz: Die Grundlagen einer Weltkarte der Meeresströmungen. Annln Hydrogr. Berl. 71, 289.

Sverdrup, H. U., Johnson, M. W. \& Fleming, R. H., 1942. The oceans, their physics, chemistry and general biology. Prentice-Hall, New York, $1087 \mathrm{pp}$.

Sorokin, J. I., 1960. Vertical distribution of phytoplankton and the primary organic production in the sea. J. Cons, perm. int. Explor. Mer 26, 49-56.

U. S. Navy Hydrographic OfFice, 1962. Provisional oceanographic charts of the tropical Atlantic ocean. Washington, D. C.

WrrtKI, K. \& BeNNETT, E. B., 1963. Vertical eddy viscosity in the Pacific equatorial undercurrent. Deep Sea Res. 10, 449-455. 